\title{
Influence of excitation energy on charged exciton formation in self-assembled InAs single quantum dots
}

\author{
E. S. Moskalenko, ${ }^{*}$ K. F. Karlsson, P. O. Holtz, and B. Monemar \\ Department of Physics and Measurement Technology, Linköping University, S-581 83 Linköping, Sweden
}

W. V. Schoenfeld, J. M. Garcia, and P. M. Petroff

Materials Department, University of California, Santa Barbara, California 93106

(Received 13 October 2000; revised manuscript received 24 January 2000; published 11 July 2001)

\begin{abstract}
We study the low-temperature photoluminescence (PL) from self-assembled InAs quantum dots as a function of a wide range of external parameters such as excitation power and pump-photon energy. By means of a conventional micro-PL setup we have succeeded in selecting the emission from a single (isolated) quantum dot. The results obtained show dramatic changes in the PL spectra initiated by changes in the pump-photon energy at a fixed excitation power: Two new lines redshifted relative to the ground-state transition appear in PL at certain pump-photon energies. This phenomenon is ascribed to the population of quantum dots with a nonequal amount of electrons and holes which is determined by the excess energies of photogenerated carriers. Based on a comparison of the spectral positions of these two lines with a simple perturbation theory model, these new lines were identified as exciton complexes charged with one and two additional electrons. It is demonstrated that the crucial role of excess energies of photogenerated carriers on the population of quantum dots with a nonequal number of electrons and holes could be used as an effective optical method to create and study charged exciton complexes in zero-dimensional semiconductor nanostructures.
\end{abstract}

DOI: 10.1103/PhysRevB.64.085302

PACS number(s): 73.21.La

\section{INTRODUCTION}

Semiconductor quantum dots (QD's) effectively confine electrons $(e)$ and holes $(h)$ on the nanometer length scale in all three directions and hence may be considered as artificial atoms with discrete energy levels for $e$ and $h$. Consequently, a QD is expected to possess zero-dimensional density of states which in turn should result in discrete and very narrow lines in the emission spectra. Indeed, such narrow lines with spectral widths down to $34 \mu \mathrm{eV}$ were observed in photoluminescence (PL) experiments. ${ }^{1}$ Another consequence originating from the more effective confinement in QD's is the increased role of Coulomb interactions with respect to the structures of higher dimensionality. ${ }^{2}$ This fact, together with the very restricted number (typically two) of particles that could be accommodated at the ground state of the QD, makes the role of multiparticle effects of great importance in a variety of aspects of the linear and nonlinear optical properties of semiconductor quantum structures. Multiparticle states consisting of a large number of $e$ and $h$ (equal or nonequal) in QD's have been studied both theoretically ${ }^{2-11}$ and experimentally. ${ }^{12-23}$

On one hand, the intensive studies in this direction could open for a number of novel physical possibilities, such as the realization of the challenging effect of Bose-Einstein condensation (BEC) of excitons in confined geometries, analogous to the discovered BEC of alkali-metal atoms in spatially confined traps ${ }^{24}$ and recently reported BEC phenomenon of indirect excitons in double-quantum-well structures, ${ }^{25}$ where excitons were spatially restricted by the heterointerface potential fluctuations, which act as "naturally grown" quantum dots. On the other hand, multiparticle effects determine the performance of a number of optoelectronic (electronic) devices, for example QD lasers and single-electron transistors, which in turn are considerably affected by the charge, stored in the QD. ${ }^{26}$ The addition of an electron to the dot results in a clear "fingerprint" in the electron addition spectra in the form of the Coulomb blockade, ${ }^{16}$ while the removal or addition of an exciton does not lead to such considerable changes due to its charge neutrality. ${ }^{7}$

This highlights the important role of multiparticle complexes with a nonequal number of $e$ and $h$, and hence stimulates the study of charged exciton complexes, which in the simplest case consist of one exciton and one additional charge carrier (i.e., $e$ or $h$ ). Also more complex charged excitons with several charge carriers of the same sign can be formed. ${ }^{8}$ For example, strong dependence of the QD emission spectra on the number of extra electrons (up to 20) was predicted in Ref. 8, where the role of the band-gap renormalization as well as the shifts of the spectral lines in the charged exciton complexes due to the exchange energy were discussed in detail. Experimental evidence for the formation of few electron states in QD's was revealed in capacitance experiments ${ }^{16,20}$ and the combined studies of capacitance and interband transmission. ${ }^{18,19}$ The formation of negatively charged exciton complexes was demonstrated in PL experiments. $^{12,17}$

However, optical studies ${ }^{17-19}$ have been based on the measurements of large ensembles of dots, so the results inevitably include the effect of inhomogeneous broadening which prevents accurate measurements and hence a detailed analysis of optical properties of the QD. That is why the studies of charged exciton phenomena in the luminescence of single dots are highly desirable. Up to date there are very few papers $^{21,22}$ that report on PL studies of charged exciton complexes in single QD's. The results obtained have allowed the identification of distinct transitions related to excitons charged with up to five additional electrons. ${ }^{21}$ It should be 
mentioned that the excess carriers in the QD's studied in Refs. 21 and 22 are due to a uniform background doping in the barriers. Hence the occupation of additional charges in the dot that occur via tunneling processes from the impurity atoms, arbitrarily positioned around the particular QD under study, is occasional and hardly controllable.

In the present contribution we propose a method to create and study charged exciton complexes in PL from a single InAs/GaAs QD. This method is based on the possibility of collecting carriers in the QD generated in the barrier ( GaAs in our samples) as a result of the optical excitation, from a rather large area (typically a few micrometer ${ }^{27}$ ) relative to the lateral size of a QD. The process of carrier diffusion depends not only on the carrier type $(e$ or $h)$, i.e. on the effective mass of the diffusing particles, but is essentially governed by the excess energy of the created carriers relative to the band gap of the excited material. The significant increase (up to five times) of the effective diffusion constant of photogenerated carriers in GaAs quantum wells with increasing pump-photon energy was experimentally demonstrated in Ref. 27. Thus it is reasonable to expect that the significantly different diffusivities of electrons and holes will lead to a population of QD's with a nonequal number of $e$ and $h$. This effect will be manifested by the appearance of charged exciton complexes in the PL spectra of individual QD's. It should be noted that opposite to the case of structures with higher dimensionalities, the emission from single QD's should be very sensitive to the extra particles because of the limited number of particles that can be captured by the QD's in total.

We here report on PL from only a single QD of InAs taking advantage of a very low density (less than $10^{7} \mathrm{~cm}^{-2}$ ) of QD's grown in combination with a conventional micro-PL ( $\mu$-PL) setup. Our results demonstrate that changes in the excitation wavelength initiate the appearance in the $\mu$-PL spectrum of two emission lines down-shifted in energy with respect to the ground-state exciton PL line. Moreover, the PL intensity of these lines as well as the intensity of the groundstate exciton shows a periodic behavior with the excitation energy (four periods are detected altogether). This circumstance together with the predicted energy shifts, based on a simple perturbation theory model, ${ }^{10}$ allows us to identify the two novel low-energy lines as being due to the exciton charged with one and two additional electrons, respectively.

\section{SAMPLES AND EXPERIMENTAL SETUP}

The samples studied were grown by molecular beam epitaxy on the semi-insulating GaAs (100) substrate. The buffer layer is prepared with a short-period superlattice 40 $\times 2 \mathrm{~nm} / 2 \mathrm{~nm}$ AlAs $/ \mathrm{GaAs}$ at a growth temperature of $630^{\circ} \mathrm{C}$. On top of a 100-nm GaAs layer the QD's are formed from a 1.7 InAs monolayer deposited at $530^{\circ} \mathrm{C}$. A first growth interruption of $30 \mathrm{sec}$ is used to improve the size distribution. Then the dots are covered with a thin GaAs cap layer with a thickness of $t_{\text {cap }}=3 \mathrm{~nm}$ before a crucial second growth interruption of $30 \mathrm{sec}$. Finally a 100-nm-thick GaAs layer is deposited to protect the QD's. Transmission electron microscopy studies of analogously grown samples revealed that uncapped original dots are lens (hemispherical) shaped with a typical lateral size of $35 \mathrm{~nm}$ and a height of $10 \mathrm{~nm} \cdot{ }^{28}$ The deposition of a GaAs capping layer after the dots have formed with $t_{\text {cap }}=3 \mathrm{~nm}$ leads to an essential reduction in the QD's height down to the $4.5 \mathrm{~nm}$. Consequently the PL is blueshifted to the spectral region of $1.34 \mathrm{eV}(925 \mathrm{~nm}),{ }^{28}$ i.e., within the sensitivity spectral range of the Si-CCD (chargecoupled device) camera. The sample was grown without rotation of the substrate, so that a gradual variation of InAs flux is achieved across the wafer, resulting in a gradient in both the density and the average size of the dots across the epitaxial layer. $^{28}$

The QD's were studied by means of a conventional diffraction-limited micro-PL setup. To excite the QD's, we used a cw-Ar laser pumped Ti-Sp laser tunable between 700 to $900 \mathrm{~nm}$ whose power was adjusted by the use of neutral filters. The beam of the Ti-Sp laser was focused on the sample surface by a microscope objective through a thin optical window of the continuous-flow cryostat. The lowest temperature available was $3.8 \mathrm{~K}$ and could be controlled with the precision of $10^{-2} \mathrm{~K}$. The laser beam could be focused on the sample surface down to a $2 \mu \mathrm{m}$ spot in diameter. The luminescence signal was collected by the same objective and dispersed by a single-grating $0.45-\mathrm{m}$ monochromator combined with a $L \mathrm{~N}_{2}$-cooled Si-CCD camera. The spectral resolution achieved in the region of the studied PL was $0.15 \mathrm{meV}$.

To find the particular QD to study, a laser beam was scanned across the sample surface. Once the desired QD was found, special marks (grids) were fabricated on the sample surface around the QD with the laser beam of very high power density. This allowed us to estimate the average distance between the adjacent QD's to be around $10 \mu \mathrm{m}$ in the studied QD structure. To control the exact position of the laser spot on the sample surface, the image of the interesting sample region was projected by a video camera, which made it easy to find the desired QD by using the fabricated marks. In addition, this arrangement allowed us to effectively correct the position of the sample if it was moved due to the thermal drift. It should be noted that with the method to locate the exact QD position with the use of the described grids one can avoid some undesirable consequences that take place with other methods. For example, when a metal mask with small holes is deposited on top of the sample, this metal mask may interact with the cap layer of the sample and acts as a stressor which could spoil the entire quality of the QD's. Four single quantum dots located at different spatial positions of the sample were examined in this study. All of them revealed an analogous behavior although their dimensions were slightly different, as reflected by a small shift in the spectral positions of the PL lines. For consistency, we present the results taken from only one single quantum dot to demonstrate a typical behavior of QD's in the present study.

\section{EXPERIMENTAL RESULTS AND DISCUSSION}

Figure 1 shows PL spectra of an individual QD taken at a temperature of $T=4 \mathrm{~K}$ and different exciting photon energies $h \nu$ in a wide range of excitation powers $P_{\mathrm{ex}}$. At low $P_{\mathrm{ex}}$ in spectra taken with an excitation energy of $h \nu=1.648 \mathrm{eV}$ 


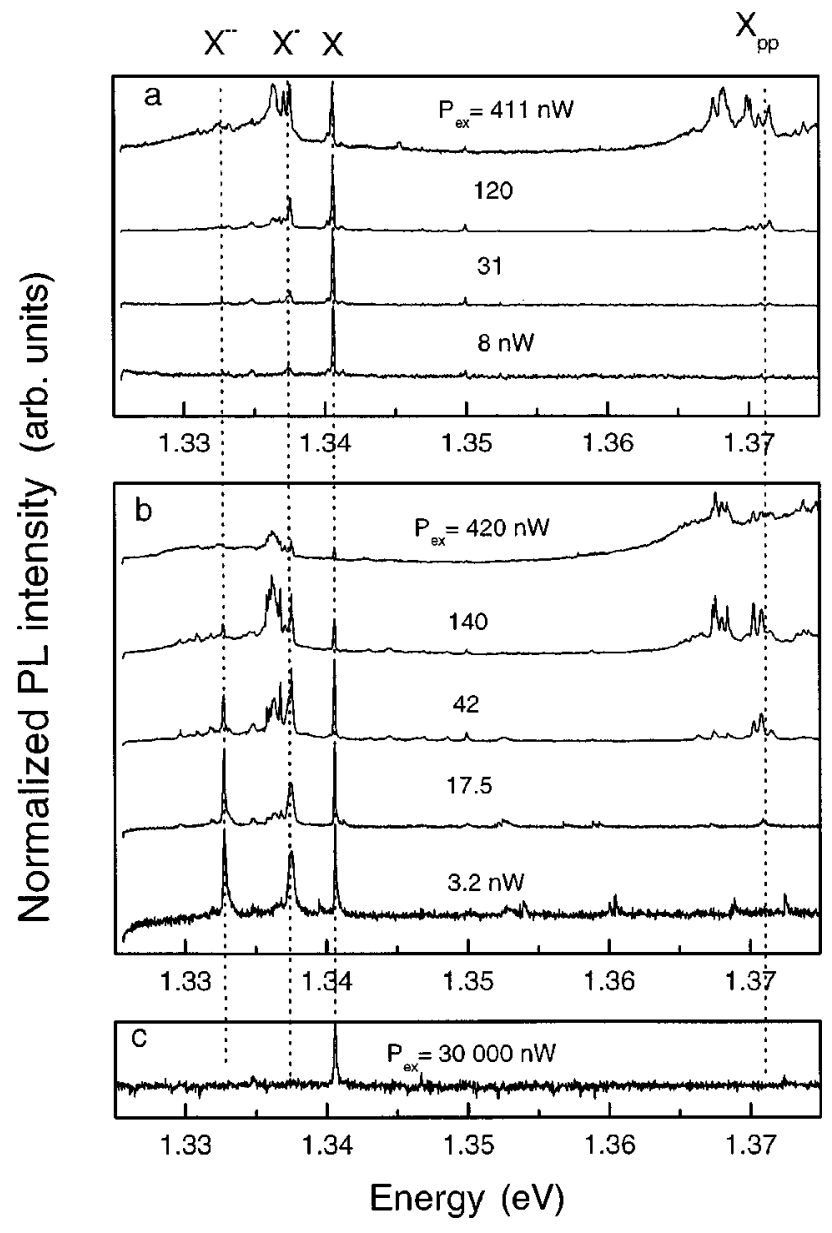

FIG. 1. PL spectra of an individual QD taken at $T=4 \mathrm{~K}$ for a number of excitation powers $P_{\text {ex }}$ (shown in the figure) and different pump-photon energies: (a) $h \nu=1.684 \mathrm{eV}$, (b) $h \nu=1.536 \mathrm{eV}$, and (c) $h \nu=1.433 \mathrm{eV}$. All spectra are normalized to the maximum value of their PL amplitude and are vertically shifted for clarity.

[Fig. 1(a)], a single line is revealed with a peak at $1.3406 \mathrm{eV}$ and labeled as $X$. This line is very narrow with its full width at half maximum of $0.15 \mathrm{meV}$, which is believed to be limited by our spectral resolution. The absence of other lines implies that the PL signal registered originates from the emission of a single QD. When increasing $P_{\text {ex }}$ up to $120 \mathrm{nW}$, two groups of lines appear in the spectrum. One group is down shifted in energy by approximately $3 \mathrm{meV}$ relative to the $X$ line, and the other group denoted as $X_{p p}$ is blueshifted by $\sim 30 \mathrm{meV}$. At further increase of $P_{\mathrm{ex}}$, the line $X$, which shows a linear dependence on $P_{\text {ex }}$ for low pump intensities, saturates and decreases its amplitude, while the intensity of the blueshifted $X_{p p}$ lines grows quadratically with $P_{\mathrm{ex}}$ and these lines dominate in PL spectra for $P_{\mathrm{ex}}>500 \mathrm{nW}$. With increasing $T$, the intensity of the $X_{p p}$ lines gradually decrease to disappear at $T>60 \mathrm{~K}$, while the line $X$ and the lines peaked in close vicinity (3-4 meV) around $X$ are still present in the PL spectra. These characteristic temperature and excitation power dependencies allow us to ascribe the lines $X_{p p}$ as the emission from the first excited state (both $e$ and $h$ occupy the corresponding $p$ level) of the QD while the lowenergy lines (around $1.34 \mathrm{eV}$ ) should be regarded as transi- tions related to the $e$ and $h$ from the lowest $s$ levels. Bayer et al. ${ }^{12}$ also observed the appearance of a similar group of lines, redshifted by 3-4 meV with respect to the $X$ line, which they interpreted in terms of biexcitonic emissions.

At another exciting photon energy $h \nu=1.536 \mathrm{eV}$ [Fig. 1(b)], the PL spectra exhibit an analogous behavior at high $P_{\text {ex }}$ while for low $P_{\text {ex }}<40 \mathrm{nW}$, dramatic differences are detected. Indeed, the spectrum taken at $P_{\mathrm{ex}}=3.2 \mathrm{nW}$ [Fig. 1(b)] clearly shows the appearance of two lines (in addition to the line $X$ ) peaked at $1.3375 \mathrm{eV}$ and $1.3328 \mathrm{eV}$ and marked as $X^{-}$and $X^{--}$correspondingly. This is in sharp contrast to the results exhibited in Fig. 1(a) obtained at another excitation energy and low $P_{\mathrm{ex}}$. To understand the dramatic changes in PL initiated by the tuning of the excitation energy, we note first that the difference occurs at lowest excitation intensity levels, so this allows us to exclude the possible influence of multiexcitonic complexes related to the occupation of QD's with more than one exciton ( $e-h$ pair) as an explanation. Second, the appearance of the $X^{-}$and $X^{--}$lines cannot be explained as an impurity related transitions, i.e., an exciton complex consisting of an optically created exciton together with a carrier (positive or negative), which is trapped by the QD from the impurity atom located in the close vicinity of the QD. In that case, the QD could be populated with an exciton irrespective of the excitation conditions, and hence the lines $X^{-}$and $X^{--}$would appear at any excitation energy (any value of $h \nu$ ). Third, both excitation energies used $h \nu$ $=1.536 \mathrm{eV}$ and $h \nu=1.648 \mathrm{eV}$ are above the GaAs barrier and hence, the observed changes in PL spectra cannot be understood in terms of the different influence of the below and above barrier excitations on the QD luminescence as was reported earlier. ${ }^{22}$ In the present study we concentrate only on the lowest $P_{\text {ex }}$ limit, at which the most dramatic differences in PL spectra are revealed when changing $h \nu$.

The evident difference for the two excitation energies used in case of Figs. 1(a) and 1(b) could be the different excess energies (with respect to the band gap) of electrons, $\Delta E_{e}$, and holes, $\Delta E_{h}$, photoexcited in the GaAs barrier. A carrier can release its excess energy via the emission of optical or acoustic phonons. If $\Delta E_{e}\left(\Delta E_{h}\right)$ is larger than the optical-phonon energy (for example, longitudional-optical phonon $\hbar \omega_{\mathrm{LO}}=36 \mathrm{meV}$ in $\mathrm{GaAs}^{27}$ ), then the particle will effectively release its energy via the cascade emission of LO phonons. These processes are very fast (of the order of a few $\mathrm{ps}^{27}$ ) and hence the "cooling" time for the particle, for example $e$, will be determined by the residual excess energy $\Delta E_{e}^{r}=\Delta E_{e}-n \times \hbar \omega_{\mathrm{LO}}$, where $n=0,1,2, \ldots$. The latter can be released only via the emission of acoustic phonons which takes much longer time. Hence the higher the $\Delta E_{e}^{r}\left(\Delta E_{n}^{r}\right)$ is, the longer time is required for $e(h)$ to cool down, form the exciton, and recombine. This eventually leads to the conclusion that particles with larger $\Delta E^{r}$ are able to move a longer distance before the exciton is formed, or in other words, they possess higher effective diffusivities. This corresponds to the experimental observation of increased effective diffusivities of carriers in GaAs quantum wells with increasing pumpphoton energy. ${ }^{27}$ Also an oscillating behavior of the photoconductivity signal, which in turn reflects the carrier mobility 


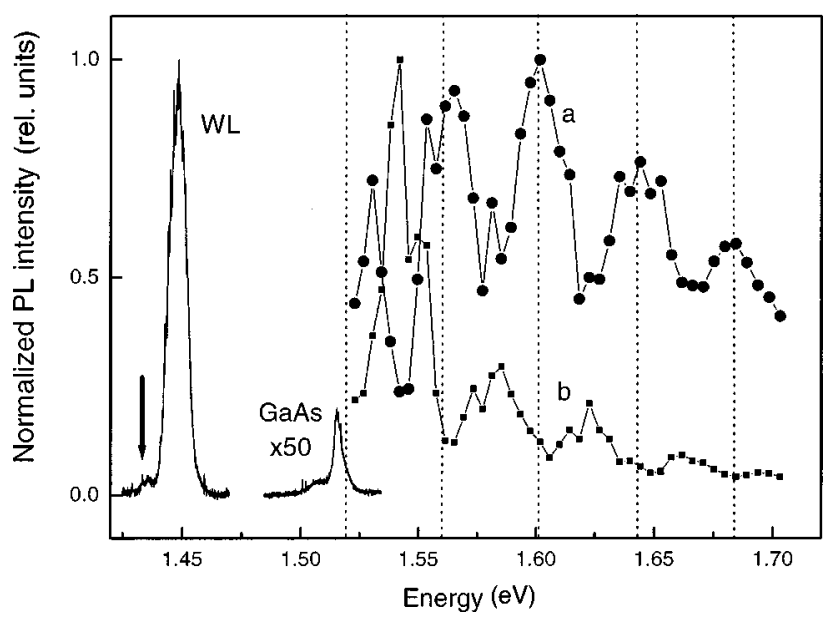

FIG. 2. The peak PL intensities of (a) $X$ and (b) $X^{--}$lines normalized to their maximum value as a function of pump-photon energy, taken at $T=4 \mathrm{~K}$ and $P_{\mathrm{ex}}=10 \mathrm{nW}$. The normalized PL spectrum of the wetting layer (WL) and of the GaAs barrier is also shown for the same values of $T$ and $P_{\text {ex }}$ at $h \nu=1.536 \mathrm{eV}$. The vertical arrow indicates the exact pump-photon energy used to obtain the PL spectrum shown in Fig. 1(c). Five vertical dotted lines separated in energy by $41.4 \mathrm{meV}$ mark the pump-photon energies at which residual electron energy (see text) is supposed to acquire equal values corresponding to that of excitation near the band gap of GaAs which is marked by the left vertical dotted line.

and hence the diffusivity, has been measured in bulk GaAs, ${ }^{29}$ where up to 15 periods were detected as the excitation energy was increased.

Experimental evidence of the importance of diffusion processes in samples studied could be obtained by comparing the intensities of the wetting layer (WL) and the GaAs emission lines peaking at 1.445 and $1.515 \mathrm{eV}$, respectively, as shown in Fig. 2. The PL intensity of the WL line is greater by a factor of 250 than that of the GaAs line although the absorption thickness of the $\mathrm{WL}$ is just $0.5 \%$ compared to the GaAs.

The difference in excess energies produced by the different $h \nu$ 's as well as the large difference in effective masses of $e$ and $h$ would imply that the numbers of $e$ and $h$ in the space region of the QD should be nonequal due to the essentially different diffusivities of the photoexcited carriers. Then it is reasonable to explain the three lines observed at low $P_{\mathrm{ex}}$ in Fig. 1(b) in terms of luminescence originating from different possible exciton configurations including "pure" exciton transition $(e-h)$ and other configurations including exciton, which has captured extra particles ( $e$ or $h$, one or several, i.e., charged excitons). One of these three observed lines [Fig. 1(b)] corresponds to the single exciton $(e-h)$. In order to find out which line corresponds to the latter transition, the QD was excited with $h \nu=1.433 \mathrm{eV}$ which corresponds to excitation resonant with the localized states of the WL. This excitation energy is marked by a thick vertical arrow in Fig. 2 and the corresponding PL spectra of the QD is shown in Fig. 1(c). In order to monitor it, an extremely high $P_{\mathrm{ex}}$ $=30000 \mathrm{nW}$ was needed due to the very low density of states in the tail of the WL emission as compared to the bulk density of states in case of the excitation above the GaAs band gap. At these excitation conditions no diffusion takes place and hence the QD is expected to be occupied with an equal number of $e$ and $h$. Indeed, we detect just a single line [Fig. 1(c)] which we ascribe to be the single exciton $(e-h)$, marked as $X$ in Fig. 1. Consequently, the remaining two lines, marked as $X^{-}$and $X^{--}$are originating from configurations when the exciton $(e-h)$ has captured extra charge(s).

We will next discuss which type of carrier $(e$ or $h)$ an exciton can bind in the case of lens- (hemispherical) shaped QD's studied here. The problem of creation of charged exciton complexes, e.g., $(2 e-h)$ or $(e-2 h)$, was considered in Ref. 9 for both types of extra carriers and for two QD's spatial profiles, hemispherical and cone shaped. The results of the detailed calculations show that in the case of hemispherical-shaped QD's, an electron is bound to the exciton, while the opposite situation takes place for the coneshaped QD's. The exact calculations of the ground-state energies ${ }^{8}$ predicted the existence of negatively charged excitons in lens-shaped QD's for the complexes consisting of one exciton and a number (up to 20) of additional electrons. By comparing the results of the detailed calculations with the results of a perturbation theory model, ${ }^{8}$ the conclusion was drawn that the exchange energy between the additional electrons and the "excitonic" electron gives the major contribution to the redshift of charged exciton complexes when the number of additional electrons increases.

On the basis of these theoretical predictions, ${ }^{8,9}$ we ascribe the lines marked as $X^{-}$and $X^{--}$to the charged exciton complexes with one and two additional electrons, respectively. Consequently, some characteristic energies, such as the binding energy of the single charged exciton $E_{\left(X^{-}\right)}^{b}$ and the exchange energy $E_{s p}^{\text {ex }}$ (between $s$ and $p$ electrons with parallel spins, as described below) can be deduced from the experiment: $E_{\left(X^{-}\right)}^{b}=E_{(X)}-E_{\left(X^{-}\right)}=3.1 \mathrm{meV}$ and $E_{s p}^{\mathrm{ex}}=E_{\left(X^{-}\right)}-E_{\left(X^{--}\right)}=4.7 \mathrm{meV}$, where $E_{(X)}, E_{\left(X^{-}\right)}$, and $E_{\left(X^{--}\right)}$correspond to the spectral positions of the $X, X^{-}$, and $X^{--}$lines, respectively. To compare these experimentally obtained values with theoretical predictions we use the simple model developed in ${ }^{10}$ to model the Coulomb blockade effect in capacitance measurements of InAs lens-shaped QD's. Lens-shaped QD's can be modeled with a parabolic confining potential ${ }^{11}$ that results in equidistant energy levels for $e$ and $h$ with energies of $\hbar \omega_{e}$ and $\hbar \omega_{h}$, respectively. In lens-shaped QD's of InAs, these energy quantities differ to a good approximation by a factor of two in favor of $\hbar \omega_{e} \cdot{ }^{10}$

In the model, the formation process of the single charged exciton can be understood as follows. When an extra electron is added to the QD, which ground state (ss) is already occupied with a single exciton, two additional interactions will appear, namely an attractive interaction with the hole $E_{s s}^{\text {attr }}$ and a repulsive interaction between the two electrons $E_{s s}^{\text {rep }}$. The competition between these two terms will determine whether the extra electron will be bound or not. In the case of $X^{--}$, a third electron is added to the QD which is already occupied with two $s$ electrons and one $s$ hole. This electron could be added only to the $p$ state in order to minimize the total energy. If this $p$ electron has its spin parallel to that of the "excitonic" electron, the $X^{--}$line will be redshifted 
relative to the $X^{-}$line by the exchange interaction energy $E_{s p}^{\mathrm{ex}}$. Following Ref. 10, the energies of interest can be written in the form

$$
\begin{gathered}
E_{s s}^{\mathrm{rep}}=e^{2} /\left[4(2 \pi)^{1 / 2} \varepsilon_{0} \varepsilon_{r} l_{e}\right], \\
E_{s s}^{\mathrm{attr}}=e^{2} /\left[4(g p)^{1 / 2} \varepsilon_{0} \varepsilon_{r}\left(l_{e}^{2}+l_{h}^{2}\right)^{1 / 2}\right], \quad E_{s p}^{\mathrm{ex}}=E_{s s}^{\mathrm{rep}} / 4,
\end{gathered}
$$

where $e$ is the elementary charge, $\varepsilon_{0}$ stands for the permittivity of vacuum, $\varepsilon_{r}=12.6$ is the dielectric constant, ${ }^{10}$ and $l_{e}$ $\left(l_{h}\right)$ stands for the electron (hole) effective length,

$$
l_{e}=\left(\hbar / m_{e}^{*} \omega_{e}\right)^{1 / 2}, \quad l_{h}=\left(\hbar / m_{h}^{*} \omega_{h}\right)^{1 / 2} .
$$

To calculate the characteristic energies we take the values of the electron and hole effective masses to be $m_{e}^{*}=0.07 m_{0}$ and $m_{h}^{*}=0.25 m_{0}\left(m_{0}\right.$ is the free-electron mass $)$, as experimentally evaluated ${ }^{10}$ for similar InAs/GaAs quantum dots used in the present study. We estimate $\hbar \omega_{e}$ and $\hbar \omega_{h}$ to be $\sim 20$ and $\sim 10 \mathrm{meV}$, respectively, using the experimentally obtained energy difference of $\sim 30 \mathrm{meV}$ between the $p p$ and $s s$ transitions ${ }^{30}$ [Figs. 1(a) and 1(b)]. Then by combining Eqs. (1) and (2) we get $E_{\left(X^{-}\right)}^{b}=E_{s s}^{\text {attr }}-E_{s s}^{\text {rep }}=2.6 \mathrm{meV}$ and $E_{s p}^{\text {(ex) }}$ $=4.9 \mathrm{meV}$, which are in good agreement with the values of 3.1 and $4.7 \mathrm{meV}$ as derived from the PL spectra. For a comparison, we studied another QD with a different size, for which $E_{p p}-E_{s s}=45 \mathrm{meV}$, revealing energy differences $E_{\left(X^{-}\right)}^{b}=3.5 \mathrm{meV}$ and $E_{s p}^{(\mathrm{ex})}=6.2 \mathrm{meV}$, while according to the above-mentioned calculation procedure corresponding values of 3.0 and $5.7 \mathrm{meV}$ were predicted.

This fact strongly supports the assignment of the $X^{-}$and $X^{--}$lines done above, and implies that at an excitation with $h \nu=1.536 \mathrm{eV}$ [Fig. 1(b)], the QD is populated with additional electrons, while in the case of pumping with $h \nu$ $=1.648 \mathrm{eV}$ [Fig. 1(a)] there are no extra electrons captured to the dot. We attribute these differences to the different excess energies of electrons at these two excitations. To further elucidate the role of electron excess energy, the dependence of the PL spectra on the pump-photon energies $h \nu$ was investigated. Figure 2 shows the evolution of the PL peak amplitude of $X$ [Fig. 2(a)] and $X^{--}$[Fig. 2(b)] lines as a function of $h \nu$. The oscillating behavior of both $X$ and $X^{--}$is clearly seen. It is important to note that the amplitudes of $X$ and $X^{--}$behave in a reversed way, i.e., when $X$ gains its maximum value, $X^{--}$acquires its minimum amplitude and vice versa. This behavior is in full agreement with our model. Indeed, every time the QD is populated with extra electrons, the creation probability (and hence the PL amplitude) of the $X^{--}$line increases, while the corresponding probability for $X$ decreases.

This picture should repeat itself whenever the electron acquires the same residual excess energy. The latter, in turn, takes place every time the pump-photon changes its energy by the value of $\Delta h \nu=\hbar \omega_{\mathrm{LO}} \times\left(1+m_{e}^{*} / m_{h}^{*}\right)$ which follows from the simple picture of band-to-band excitation in direct band-gap semiconductors in the approximation of parabolic valence and conduction bands. Taking the electron and heavy-hole effective masses in GaAs as $m_{e}^{*}=0.067 m_{0}$ and $m_{h}^{*}=0.45 m_{0}{ }^{27}$ and $\hbar \omega_{\mathrm{LO}}=36 \mathrm{meV}$, we obtain $\Delta h \nu$ $=41.4 \mathrm{meV}$. Five vertical dotted lines in Fig. 2 are plotted with this energy separation starting from the value of 1.519 $\mathrm{eV}$, which corresponds to the band gap of bulk GaAs. It is seen that they intersect characteristic minimum (maximum) amplitude values of the $X^{--}(X)$ lines. This circumstance, in turn, is consistent with our model, that it is the electrons which initiate the dramatic changes observed in PL spectra when tuning the excitation photon energy.

Finally, it should be mentioned that the amplitude of the line $X^{-}$also shows an oscillating behavior with the excitation energy, although it is not so clear as in the case of the lines $X$ and $X^{--}$. This fact could be qualitatively understood on the basis of the following considerations: When $h \nu$ is changed, the probability of capturing an extra electron to the QD is changed according to our model. If the capture probability increases, the amplitude of line $X^{-}$should increase, while correspondingly, the intensity of line $X$ will decrease. At the same time, some redistribution of the intensities of the lines $X^{-}$and $X^{--}$in favor of the line $X^{--}$should also take place, which gives rise to a decrease of the $X^{-}$line amplitude. Due to these competing processes, the analysis of the $X^{-}$line amplitude behavior with $h \nu$ is more complicated and less evident compared to the case of the line $X\left(X^{--}\right)$, which clearly decreases (increases) its amplitude in this particular case.

\section{CONCLUSION}

In summary, the PL spectra from single InAs QD's have been investigated as a function of the excitation power and the pump-photon energy. For a fixed excitation power, the PL spectra show dramatic changes as a function of excitation energy: For certain pump-photon energies, two new excitonic lines appear that are redshifted relative to the single exciton. A qualitative model is proposed, which predicts the crucial role of excess energy of the photogenerated electrons and explains the oscillating behavior of the PL amplitudes observed in the experiment. This fact together with estimates made by means of a simple perturbation theory model ${ }^{10}$ allowed us to identify all three lines observed in PL at low $P_{\mathrm{ex}}$ : the $X^{-}$and $X^{--}$lines are interpreted as excitons charged with one and two additional electrons, respectively. The pump-photon energy influence on PL spectra is proposed as a new interesting method to create charged exciton complexes in quantum dots in a controlled way.

\section{ACKNOWLEDGMENTS}

One of us (E.S.M.) gratefully acknowledges financial support of Swedish Institute within the Visby Programme. 
*Permanent address: A. F. Ioffe Physical-Technical Institute, Russian Academy of Sciences, 194021, Polytechnicheskaya 26, St. Petersburg, Russia.

${ }^{1}$ H. Kamada, H. Ando, J. Temmyo, and T. Tammamura, Phys. Rev. B 54, 16243 (1998).

${ }^{2}$ G. Medeiros-Ribeiro, F. G. Pikus, P. M. Petroff, and A. L. Efros, Phys. Rev. B 55, 1568 (1997).

${ }^{3}$ S. Raymond, P. Hawrylak, C. Gould, S. Fafard, A. Sachrajda, M. Potemski, A. Wojs, S. Charbonneau, D. Leonard, P. M. Petroff, and J. L. Merz, Solid State Commun. 101, 883 (1997).

${ }^{4}$ S. Bednarek, B. Szafran, and J. Adamowski, Phys. Rev. B 59, 13036 (1999).

${ }^{5}$ M. Rontani, F. Rossi, F. Manghi, and E. Molinari, Phys. Rev. B 59, 10165 (1999).

${ }^{6}$ E. Dekel, D. Gershoni, E. Ehrenfreund, J. M. Garcia, and P. M. Petroff, Phys. Rev. B 61, 11009 (2000).

${ }^{7}$ P. Hawrylak, Phys. Rev. B 60, 5597 (1999).

${ }^{8}$ A. Wojs and P. Hawrylak, Phys. Rev. B 55, 13066 (1997).

${ }^{9} \mathrm{Ph}$. Lelong and G. Bastard, Solid State Commun. 98, 819 (1996).

${ }^{10}$ R. J. Warburton, B. T. Miller, C. S. Durr, C. Bodefeld, K. Karrai, J. P. Kotthaus, G. Medeiros-Ribeiro, P. M. Petroff, and S. Huant, Phys. Rev. B 58, 16221 (1998).

${ }^{11}$ A. Wojs and P. Hawrylak, Phys. Rev. B 53, 10841 (1996); 51, 10880 (1995).

${ }^{12}$ M. Bayer, T. Gutbrod, A. Forchel, V. D. Kulakovskii, A. Gorbunov, M. Michel, R. Steffen, and K. H. Wang, Phys. Rev. B 58, 4740 (1998); M. Bayer, A. Kuther, F. Schafer, J. P. Reithmaier, and A. Forchel, ibid. 60, R8481 (1999).

${ }^{13}$ E. Dekel, D. Gershoni, E. Ehrenfreund, D. Spector, J. M. Garcia, and P. M. Petroff, Phys. Rev. Lett. 80, 4991 (1998).

${ }^{14}$ K. Brunner, G. Abstreiter, G. Bohm, G. Trankle and G. Weinmann, Phys. Rev. Lett. 73, 113814 (1994).

${ }^{15}$ L. Landin, M. E. Pistol, C. Pryor, L. Samuelson, and M. Miller, Phys. Rev. B 60, 16640 (1999).

${ }^{16}$ S. Tarucha, D. G. Austing, T. Honda, R. J. van der Hage, and L. P. Kouwenhoven, Phys. Rev. Lett. 77, 3613 (1996).

${ }^{17}$ K. H. Schmidt, G. Medeiros-Ribeiro, M. Oestreich, P. M. Petroff, and G. H. Dohler, Phys. Rev. B 54, 11346 (1996); 58, 3597 (1998).

${ }^{18}$ H. Drexler, D. Leonard, W. Hansen, J. P. Kotthaus and P. M.
Petroff, Phys. Rev. Lett. 73, 2252 (1994).

${ }^{19}$ R. J. Warburton, C. S. Durr, K. Karrai, J. P. Kotthaus, G. Medeiros-Ribeiro, and P. M. Petroff, Phys. Rev. Lett. 79, 5282 (1997).

${ }^{20}$ B. T. Miller, W. Hansen, S. Manus, R. J. Luyken, A. Lorke, J. P. Kotthaus, and S. Huant, Phys. Rev. B 56, 6764 (1997).

${ }^{21}$ A. Hartmann, Y. Ducommun, E. Kapon, U. Hohenester, and E. Molinari, Phys. Rev. Lett. 84, 5648 (2000).

${ }^{22}$ A. Hartmann, Y. Ducommun, M. Bachthold, and E. Kapon, Physica E 7, 461 (2000).

${ }^{23}$ P. Paskov, P. O. Holtz, S. Wongmanerod, B. Monemar, J. M. Garcia, W. V. Schoenfeld, and P. M. Petroff, Physica E 6, 440 (2000).

${ }^{24}$ F. Dalfolo, S. Giorgini, L. P. Pitaevskii, and S. Stringari, Rev. Mod. Phys. 71, 463 (1999).

${ }^{25}$ E. S. Moskalenko, V. V. Krivolapchuk, and A. L. Zhmodikov, Fiz. Tverd. Tela (St. Petersburg) 42, 1492 (2000) [Phys. Solid State 42, 1535 (2000)].

${ }^{26}$ D. Bimberg, M. Grundmann, and N. N. Ledentsov, Quantum Dot Heterostructures (Wiley, London, 1999); T. Lundstrom, W. Schoenfeld, H. Lee, and P. M. Petroff, Science 286, 2312 (1999).

${ }^{27}$ J. P. Wolfe, H. W. Yoon, D. R. Wake, and H. Morkoc, Semicond. Sci. Technol. 7, B240 (1992); H. W. Yoon, D. R. Wake, and J. P. Wolfe, Phys. Rev. B 54, 2763 (1996).

${ }^{28}$ K. H. Schmidt, G. Medeiros-Ribeiro, J. M. Garcia, and P. M. Petroff, Appl. Phys. Lett. 70, 1727 (1997); J. M. Garcia, T. Mankad, P. O. Holtz, P. J. Wellman, and P. M. Petroff, ibid. 72, 3172 (1998); J. M. Garcia, G. Medeiros-Ribeiro, K. Schmidt, T. Ngo, J. L. Feng, A. Lorke, J. Kotthaus, and P. M. Petroff, ibid. 71, 2014 (1997).

${ }^{29}$ R. W. Shaw, Phys. Rev. B 3, 3283 (1971).

${ }^{30}$ As follows from the solution of the simple two-dimensional harmonic oscillator, the ratio $\hbar \omega_{h} / \hbar \omega_{e}$ depends only on the effective masses and the depths of the confining parabolic potentials. Therefore, $\hbar \omega_{h} / \hbar \omega_{e}$ is expected to be close to $\frac{1}{2}$ for the QD's studied in the present paper, as obtained both experimentally and theoretically for similar QD's by Refs. 10 and 31.

${ }^{31}$ A. Wojs, P. Hawrylak, S. Fafard, and L. Jacak, Phys. Rev. B 54, 5604 (1996). 